\title{
Intensive care admission and hospital mortality in the elderly after non- cardiac surgery in Spain: secondary analysis of EuSOS study
}

\author{
de Nadal M.', Pérez-Hoyos S. ', Montejo-González J.C.', Pearse R. ${ }^{3}$, Aldecoa C. ${ }^{4}$, European Surgical Outcomes Study (EuSOS) Study Group. \\ Hospital Vall d'Hebron, Barcelona, Spain', Hospital Universitario 12 de Octubre, Madrid, Spain², Barts and The London School of Medicine and Dentistry, Queen Mary \\ University of London, London, United Kingdom ${ }^{3}$ and Hospital Universitario Rio Hortega, Valladolid, Spain ${ }^{4}$
}

Background and Goal of Study: Post-operative intensive care admission may improve outcomes, especially in high-risk patients. Very elderly patients (over 80 or 85 years) represent a high-risk population due to their frailty and associated morbidity. The findings of studies exploring the benefits of post-operative intensive care admission for elderly patients are inconsistent. The aim of this study was to determine the impact of age as an independent factor of ICU admission after non-cardiac surgery in Spain using data from the European Surgical Outcome Study (EuSOS).

Materials and Methods: A descriptive analysis of the Spanish subset of the EuSOS (1), including demographic and surgical characteristics, was carried out. Age differences of ICU admission and mortality, adjusted by other demographic and surgical variables, were tested by means of a chi squared and KruskalWallis test. A logistic model was fitted to relate age with ICU admission, outcomes and mortality. A multivariate model was built adding the demographic and surgical variables.

Results: The Spanish subset of EuSOS consisted of 5,433 patients, of which 5,412 were included for analysis. A total of 677 patients (12.5\%) were admitted to an ICU after surgery. A significant relationship between age and ICU admission was found, so that for each year the OR likelihood for ICU admission increased 1.02. In the fractional polynomial regression analysis, this effect disappeared after adjusting for the other variables, with a significant decrease in the probability of admission from 80 years on.

Demographic and surgical characteristics among age categories:

\begin{tabular}{|c|c|c|c|c|c|c|c|}
\hline & & $\begin{array}{l}<65 \text { years } \\
(\mathrm{n}=3195)\end{array}$ & $\begin{array}{l}\text { 65-74 years } \\
(\mathrm{n}=1069)\end{array}$ & $\begin{array}{l}\text { 75-85 years } \\
(\mathrm{n}=959)\end{array}$ & $\begin{array}{l}>85 \text { years } \\
(\mathrm{n}=189)\end{array}$ & $\begin{array}{l}\text { Total } \\
(\mathrm{n}=5412)\end{array}$ & $P$ value \\
\hline Men & & $1590(49.8)$ & $472(44.1)$ & $499(52.0)$ & $126(66.7)$ & 2687 (49.6\%) & $<0.001$ \\
\hline Smoker & & $974(30.5)$ & $133(12.4)$ & $73(7.6)$ & $7(3.7)$ & $1187(21.9 \%)$ & $<0.001$ \\
\hline \multirow[t]{5}{*}{ ASA score } & I & $1043(32.6 \%)$ & $48(4.5 \%)$ & $11(1.1 \%)$ & $2(1.1 \%)$ & $1104(20.4 \%)$ & $<0.001$ \\
\hline & II & $1624(50.8 \%)$ & $600(56.1 \%)$ & $410(42.7 \%)$ & $49(25.9 \%)$ & $2683(49.6 \%)$ & \\
\hline & III & $462(14.5 \%)$ & $378(35.4 \%)$ & $440(45.9 \%)$ & $108(57.1 \%)$ & $1388(25.6 \%)$ & \\
\hline & IV & $62(1.9 \%)$ & $40(3.7 \%)$ & $96(10 \%)$ & $29(15.3 \%)$ & $227(4.2 \%)$ & \\
\hline & $\mathrm{v}$ & $4(0.1 \%)$ & $3(0.3 \%)$ & $2(0.2 \%)$ & $1(0.5 \%)$ & $10(0.2 \%)$ & \\
\hline \multirow[t]{8}{*}{ Comorbidities } & Cirrhosis & $54(1.7 \%)$ & $17(1.6 \%)$ & $10(1.0 \%)$ & $2(1.1 \%)$ & $83(1.5 \%)$ & 0.502 \\
\hline & $\mathrm{CHF}$ & $30(0.9 \%)$ & $39(3.6 \%)$ & $76(7.9 \%)$ & $23(12.2 \%)$ & $168(3.1 \%)$ & $<0.001$ \\
\hline & COPD & $261(8.1 \%)$ & $159(14.9 \%)$ & $161(16.8 \%)$ & $31(16.4 \%)$ & $612(11.3 \%)$ & $<0.001$ \\
\hline & CAD & $97(3.0 \%)$ & $128(12 \%)$ & $161(16.8 \%)$ & $33(17.5 \%)$ & $419(7.7 \%)$ & $<0.001$ \\
\hline & Diabetes (insulin) & $85(2.7 \%)$ & $69(6.4 \%)$ & $90(9.4 \%)$ & $10(5.3 \%)$ & $254(4.7 \%)$ & $<0.001$ \\
\hline & Diabetes (no & $177(5.5 \%)$ & $147(13.7 \%)$ & $188(19.6 \%)$ & $34(18 \%)$ & $546(10.1 \%)$ & $<0.001$ \\
\hline & Metastatic disease & $119(3.7 \%)$ & $62(5.8 \%)$ & $41(4.3 \%)$ & $6(3.2 \%)$ & $228(4.2 \%)$ & 0.028 \\
\hline & Stroke & $55(1.7 \%)$ & $67(6.3 \%)$ & $101(10.5 \%)$ & $20(10.6 \%)$ & $243(4.5 \%)$ & $<0.001$ \\
\hline \multirow[t]{3}{*}{ Grade of surgery } & Minor & $629(19.7 \%)$ & $169(15.8 \%)$ & $129(13.4 \%)$ & $23(12.2 \%)$ & $950(17.5 \%)$ & $<0.001$ \\
\hline & Intermediate & $1652(51.7 \%)$ & $481(45 \%)$ & 445 (46.4\%) & $109(57.7 \%)$ & 2687 (49.6\%) & \\
\hline & Major & $914(28.6 \%)$ & 419 (39.2\%) & $385(40.1 \%)$ & $57(30.2 \%)$ & $1775(32.8 \%)$ & \\
\hline \multirow[t]{3}{*}{ Urgency of surgery } & Elective & $2636(82.5 \%)$ & $929(86.9 \%)$ & $754(78.6 \%)$ & $117(61.9 \%)$ & $4436(82 \%)$ & $<0.001$ \\
\hline & Urgent & $491(15.4 \%)$ & $128(12 \%)$ & $180(18.8 \%)$ & $65(34.4 \%)$ & $864(16 \%)$ & \\
\hline & Emergent & $68(2.1 \%)$ & $12(1.1 \%)$ & $25(2.6 \%)$ & $7(3.7 \%)$ & $112(2.1 \%)$ & \\
\hline \multirow[t]{12}{*}{ Surgical speciality } & Orthopaedics & $766(24 \%)$ & $268(25.1 \%)$ & $312(32.5 \%)$ & $80(42.3 \%)$ & $1426(26.3 \%)$ & $<0.001$ \\
\hline & Breast & $111(3.5 \%)$ & $32(3 \%)$ & $17(1.8 \%)$ & $3(1.6 \%)$ & $163(3 \%)$ & \\
\hline & Gynaecology & $338(10.6 \%)$ & $52(4.9 \%)$ & $46(4.8 \%)$ & $7(3.7 \%)$ & $443(8.2 \%)$ & \\
\hline & Vascular & $122(3.8 \%)$ & $58(5.4 \%)$ & $81(8.4 \%)$ & $24(12.7 \%)$ & $285(5.3 \%)$ & \\
\hline & Upper GI & $129(4.0 \%)$ & $60(5.6 \%)$ & $44(4.6 \%)$ & $6(3.2 \%)$ & $239(4.4 \%)$ & \\
\hline & Lower GI & $381(11.9 \%)$ & $125(11.7 \%)$ & $137(14.3 \%)$ & $22(11.6 \%)$ & $665(12.3 \%)$ & \\
\hline & Hepato-biliary & $185(5.8 \%)$ & $74(6.9 \%)$ & $48(5.0 \%)$ & $6(3.2 \%)$ & $313(5.8 \%)$ & \\
\hline & Plastic & $164(5.1 \%)$ & $32(3 \%)$ & $28(2.9 \%)$ & $7(3.7 \%)$ & $231(4.3 \%)$ & \\
\hline & Urology & $272(8.5 \%)$ & $202(18.9 \%)$ & $131(13.6 \%)$ & $18(9.5 \%)$ & $623(11.5 \%)$ & \\
\hline & Kidney & $30(0.9 \%)$ & $15(1.4 \%)$ & $4(.04 \%)$ & $1(0.5 \%)$ & $50(0.9 \%)$ & \\
\hline & Head and Neck & $506(15.8 \%)$ & $85(7.9 \%)$ & $50(5.2 \%)$ & $7(3.7 \%)$ & $648(12 \%)$ & \\
\hline & Other & $191(6 \%)$ & $66(6.2 \%)$ & $61(6.3 \%)$ & $8(4.2 \%)$ & $326(6.0 \%)$ & \\
\hline
\end{tabular}

Relation between age and intensive care admission in a fractional polynomial model $(-2,2)$.

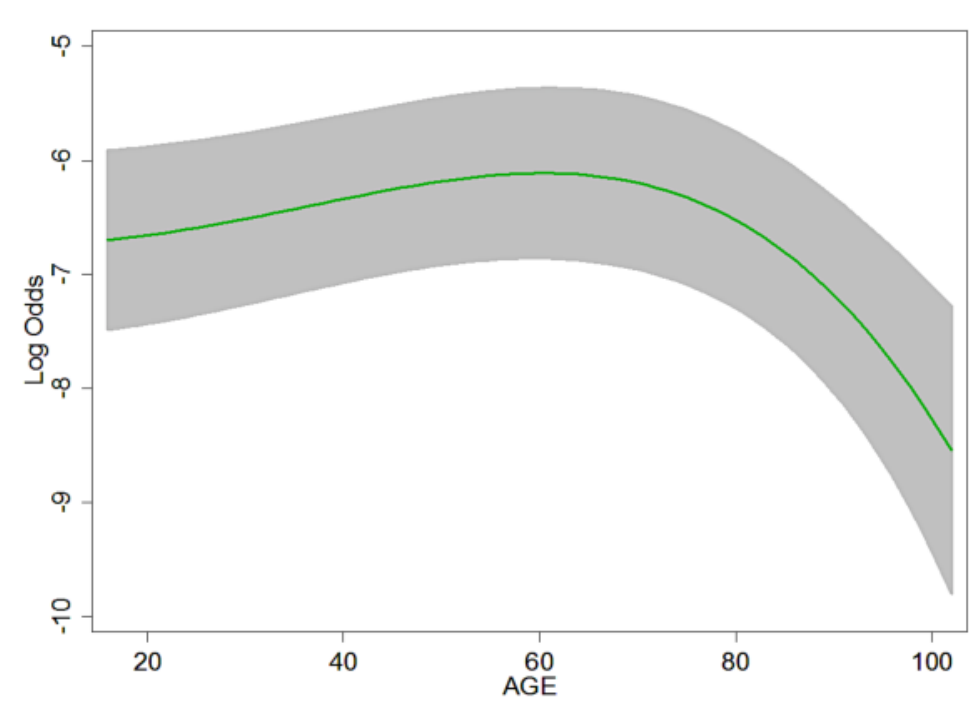

Independently associated factors for ICU admission:

\begin{tabular}{|c|c|c|c|c|}
\hline & & $\begin{array}{l}\text { ICU } \\
\text { admission }\end{array}$ & OR $(95 \% \mathrm{CI})$ & P valuc \\
\hline \multirow[t]{4}{*}{ Age } & $<65$ years & $339(10.6)$ & 1 & 0.004 \\
\hline & 65-74 years & $181(16.8)$ & $1.08(0.84-1.39)$ & \\
\hline & 75-85 years & $140(14.5)$ & $0.73(0.55-0.97)$ & \\
\hline & $>85$ vears & $17(9.0)$ & $0.41(0.22-0.77)$ & \\
\hline \multirow[t]{5}{*}{ ASA Score } & $\mathrm{I}$ & $40(3.6)$ & 1 & $<0.001$ \\
\hline & II & $249(9.3)$ & $1.99(1.36-2.91)$ & \\
\hline & III & $284(20.4)$ & $3.30(2.19-4.98)$ & \\
\hline & IV & $96(42.1)$ & $8.99(5.23-15.45)$ & \\
\hline & $\mathrm{V}$ & $8(72.7)$ & $10.02(1.56-64.42)$ & \\
\hline \multirow[t]{3}{*}{ Grade of surgery } & Minor & $12(1.3)$ & 1 & $<0.001$ \\
\hline & Intermediate & $122(4.5)$ & $3.76(2.05-6.91)$ & \\
\hline & Maior & $543(30.5)$ & $30.93(17.10-55.95)$ & \\
\hline \multirow[t]{3}{*}{ Urgency of surgery } & Elective & $507(11.4)$ & 1 & $<0.001$ \\
\hline & Urgent & $117(13.5)$ & $1.06(0.80-1.40)$ & \\
\hline & Emergencv & $53(46.9)$ & $4.22(2.44-7.29)$ & \\
\hline \multirow[t]{12}{*}{ Surgical speciality } & Orthopaedics & $71(4.9)$ & 1 & $<0.001$ \\
\hline & Breast & $17(10.4)$ & $3.18(1.71-5.91)$ & \\
\hline & Gynaecology & $26(5.9)$ & $1.98(1.19-3.32)$ & \\
\hline & Vascular & $78(27.3)$ & $5.48(3.59-8.32)$ & \\
\hline & Unper gastrointestinal & $76(31.8)$ & $7.39(4.85-11.33)$ & \\
\hline & Lower gastrointestinal & $131(19.7)$ & $4,22(2.99-6.05)$ & \\
\hline & Hepato-biliary & $64(20.4)$ & $5.54(3.55-8.67)$ & \\
\hline & Plastic & $11(4.7)$ & $2.76(1.36-5.63)$ & \\
\hline & Urology & $63(10.1)$ & $2.74(1.83-4.08)$ & \\
\hline & Kidnev & $18(36)$ & $4.83(2.36-9.89)$ & \\
\hline & Head and Neck & $55(8.5)$ & $3.49(2.32-5.25)$ & \\
\hline & Other & $62(19)$ & $5.29(3.45 ; 8.10)$ & \\
\hline Metastasic disease & & $86(37.4)$ & $2.28(1.61-3.23)$ & $<0.001$ \\
\hline Laparoscopic surgery & & $84(12.4 \%)$ & $0.68(0.50 ; 0.92)$ & 0.012 \\
\hline
\end{tabular}

Independently associated factors for hospital mortality:

\begin{tabular}{|c|c|c|c|c|}
\hline & & $\begin{array}{l}\text { Hospital } \\
\text { mortality }\end{array}$ & OR $(95 \% \mathrm{CI})$ & P value \\
\hline \multirow[t]{5}{*}{ ASA Score } & I & $45(4.0 \%)$ & 1 & $<0.001$ \\
\hline & II & $70(2.6 \%)$ & $0.59(0.39-0.90)$ & \\
\hline & III & $53(3.8 \%)$ & $0,71(0.42-1.20)$ & \\
\hline & IV & $32(14.0 \%)$ & $2.20(1.14-4.26)$ & \\
\hline & $\mathrm{V}$ & $8(72.7 \%)$ & $17.81(3.80-83.59)$ & \\
\hline \multirow[t]{3}{*}{ Urgency of surgery } & Elective & $139(3.1 \%)$ & 1 & $<0.001$ \\
\hline & Urgent & $48(5.5 \%)$ & $1.44(0.99-2.09)$ & \\
\hline & Emergency & $21(18.6 \%)$ & $3.31(1.77-6.19)$ & \\
\hline \multirow[t]{12}{*}{ Surgical speciality } & Orthopaedics & $36(2.5 \%)$ & 1 & $<0.001$ \\
\hline & Breast & $4(2.4 \%)$ & $1.30(0.45-3.75)$ & \\
\hline & Gynaecology & $26(5.9 \%)$ & $3.29(1.88-5.76)$ & \\
\hline & Vascular & $20(7.0 \%)$ & $1.78(0.95-3.33)$ & \\
\hline & Upper gastrointestinal & $20(8.4 \%)$ & $3.45(1.88-6.33)$ & \\
\hline & Lower gastrointestinal & $33(5 \%)$ & $1.57(0.94-2.64)$ & \\
\hline & Hepato-biliarv & $14(4.5 \%)$ & $1.91(0.96-3.80)$ & \\
\hline & Plastic & $5(2.2 \%)$ & $0.96(0.37-2.51)$ & \\
\hline & Urology & $20(3.2 \%)$ & $1.46(0.81-2.62)$ & \\
\hline & Kidney & $1(2 \%)$ & $0.70(0.09-5.51)$ & \\
\hline & Head and Neck & $17(2.6 \%)$ & $1.24(0.68-2.27)$ & \\
\hline & Other & $9(2.8 \%)$ & $1.04(0.48-2.26)$ & \\
\hline Diabetis insulin-dependant & & $22(8.7 \%)$ & $2.08(1.22-3.53)$ & 0.007 \\
\hline
\end{tabular}

Conclusions: Patients aged over 80 years appear less likely to be admitted to ICU after non-cardiac surgery in Spanish hospitals. However, this lower rate of ICU admission did not affect hospital mortality, which was independent of age category. The role of PACUs or ward-care based may be associated with age and affect outcomes after surgery. Further studies are needed to clarify the safest and effective postoperative care pathway for elderly population.

References: (1) Pearse RM et al. Mortality after surgery in Europe: a 7 day cohort study. Lancet 2012 